\title{
Detection and classification real-time of fall events from the daily activities of human using forward scattering radar
}

\begin{abstract}
Detection and identification of various human activities that have concurrently performed by two individuals or more is a crucial task of elderly assisted living systems. Fall is the biggest problem which may threaten the older people's life aged 65 and above, therefore, the realtime detection of human activities and classification of fall events is required whether in their houses or in the health care institutions. This paper presents a Forward Scattering Radar as a monitoring sensor for the real-time categorizing features of falls from the non-fall activities. The spectrogram representations are utilized for analyzing motion characteristics, while, based on the short-time Fourier transform features, the support vector machine has been used for classification operations. An indoor experiment was carried out to emulate the sitting on a chair of the older and forward falling down event, where 50 trials were fulfilled by 5 adults for each activity. The analysis results indicated that the Forward Scattering Radar has a pretty good ability in detecting of the daily activities and classification of fall from the different overlapping activities. The preliminary classification results have revealed a noticeable classification performance of the fall event when the two activities, the forward falling and sitting on a chair, are happened simultaneously.
\end{abstract}

Keyword: Elderly fall detection; Forward scattering radar; Time-frequency domain analysis; Support vector machine; Real-time classification 